- Discusses complications of an inadequately or unreduced zygomatic arch fracture.

- Considers various methods used for assessment of the reduction of zygomatic arch fractures

- Describes the C-Arm technique for dynamic visualisation of instrumentation and the immediate monitoring of the adequacy of zygomatic arch fracture reduction intraoperatively.

\title{
C-Arm for accurate reduction of zygomatic arch fracture - A case report
}

\author{
S. J. Badjate ${ }^{1}$ and K. M. Cariappa ${ }^{2}$
}

Fracture of the zygomatic arch is usually treated using blind methods. As the fracture lines cannot be visualised directly in closed reduction, digital exploration and crepitus noise or conventional radiographic imaging are used clinically as a guide to reposition the fragments. Successful closed reductions are often difficult to evaluate clinically because of the great amount of swelling that often accompanies these fractures. Postoperative radiographs are often the only way to assess the adequacy of the reduction. This article describes a technique that uses the C-Arm to quickly and accurately evaluate the reduction intraoperatively so that appropriate corrections can be made. A case report of a patient who suffered a w-shaped depressed fracture of the left zygomatic arch and zygomatico-maxillary complex fracture is presented.

\section{INTRODUCTION}

Depressed zygomatic arch fractures generally implicate a partial or total obstruction of the movement of the condyle and of the coronoid process of the mandible, changing the opening and closure of the mouth. ${ }^{1}$

Numerous techniques have been described to reduce zygomatic arch fractures. Conventionally they are conducted by a blind method, because the anatomical pathways of the facial nerve rule out any large incision. Fragments are repositioned using bone elevators passed through small incisions in the temporal skin or in the mucosa of the gingival sulcus in the canine fossa. Successful reductions are often difficult to evaluate clinically because of the great amount of swelling that often accom-

\footnotetext{
1*Postgraduate student, Department of Oral and Maxillofacial Surgery, Manipal College of Dental Sciences, Manipal, India; ${ }^{2}$ Professor and Head, Department of Oral and Maxillofacial surgery, Manipal College Of Dental Sciences, Manipal, India.

${ }^{*}$ Correspondence to: Dr Samprati J. Badjate, c/o Mr L. Tonse, Indrani Temple Road, Near MSIL Depot, Green Top House, Indrali, Udupi, Karanataka 576102, India., Email:drsamprati@rediffmail.com
}

\section{Refereed Paper}

panies these fractures. ${ }^{2}$ The position of the fragment is usually confirmed by palpation and radiography during operation. However, digital exploration and crepitus are unreliable guides in some cases, conventional radiographic imaging during surgery often presents difficulties in positioning the patient, and the delay in printing the film increases the operative time.

The C-Arm can obviate the need for intraoperative radiographs that, due to technician and film processing delays, add significantly to operative time.

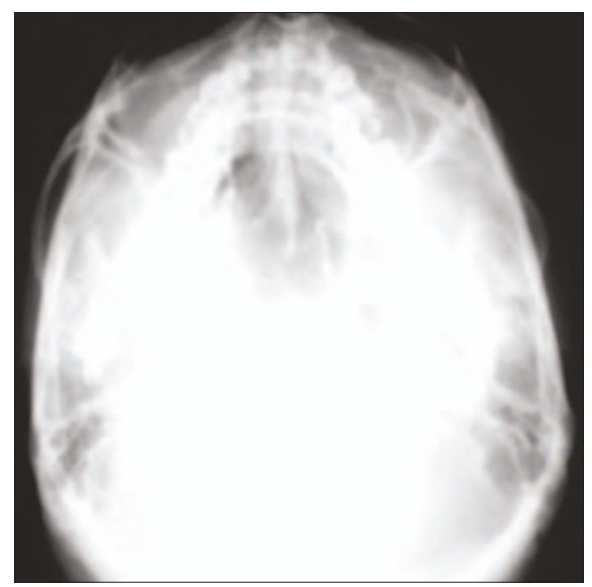

Fig. 1 Showing 'w' fracture of zygomatic arch

\section{CASE REPORT}

A 25-year-old man reported to the trauma centre of Kasturba Medical College and Hospital with a history of a road traffic accident. Clinical examination revealed a depression and marked pain in the left side of his face. Radiological examination revealed fractures of the zygomatic arch and the zygomatico-maxillary complex left side (Fig. 1). Reduction and fixation of the zygomatico-maxillary complex was performed using the miniplate system. Zygomatic arch fracture was reduced by the KILLEY technique ${ }^{2}$ which consists of a surgical reduction with access via the Gillies approach with the use of the Bristow elevator. It does not consider external fixation necessary due to the presence of the fascia of the temporal muscle.

The C-Arm was used intraoperatively to check the proper reduction of the fragments (Fig. 2). The stability of reduced fragments checked adequately. The patient was instructed to follow a soft diet for 8-10 days post-operatively to limit the use of the masseter muscle, which pulls downward. ${ }^{3}$ The patient was also advised not to apply pressure on the same side. 


\section{PRACTICE}

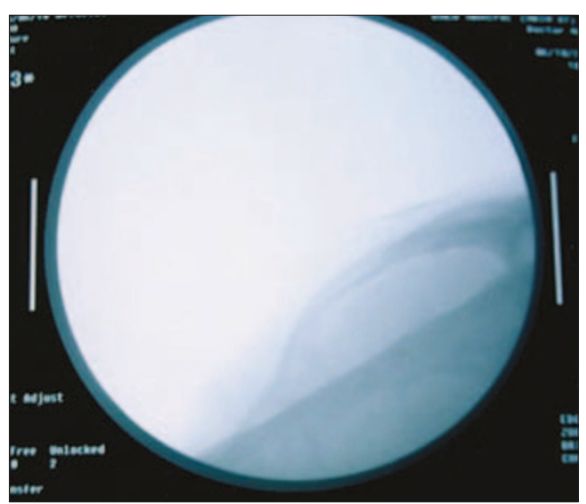

Fig. 2 Showing intraoperative C-Arm image of reduced zygomatic arch

\section{DISCUSSION}

Up until now it has only been possible to monitor the alignment of zygomatic arch fractures postoperatively with a computed tomography (CT) examination with coronal sectioning or conventional radiography. If this showed an incorrect positioning, renewed surgery and anaesthetics were often required.

Fracture of the zygomatic arch is usually treated using blind methods. Because the fracture lines cannot be visualised directly in closed reduction, digital exploration and crepitus noise or conventional radiographic imaging are used clinically as a guide to reposition the fragments. Operative methods that do not allow intraoperative visualisation of the fracture fragments not only result in unsatisfactory reduction, but are also fraught with complications including persistent diplopia, orbital dystopia, malunion, and significant residual deformity. The complications of an inadequately or unreduced zygomatic fracture are very difficult to correct secondarily and are usually avoidable. In the authors' experience, closed reductions without intraoperative assessment of reduction yield unpredictable results with a significant chance of relapse, which gives rise to an embarrassing situation for both surgeon and patient postoperatively.

Various methods like digital palpation and crepitus, radiographic imaging, Sonography ${ }^{4}$, and portable CT Scan ${ }^{5}$ are used for intraoperative assessment of reduction of zygomatic arch fractures. Digital exploration and crepitus has the disadvantage that successful reductions are often difficult to evaluate clinically because of the great amount of swelling that often accompanies these fractures. Hence, it is an unreliable guide. The radiographic-imaging technique creates difficulty in positioning the patient, high risk of exposure to radiation and adds significantly to operative time due to the technician and film processing delay. In Sonography, minimally displaced fractures are not well appreciated ${ }^{5}$ so assessment for

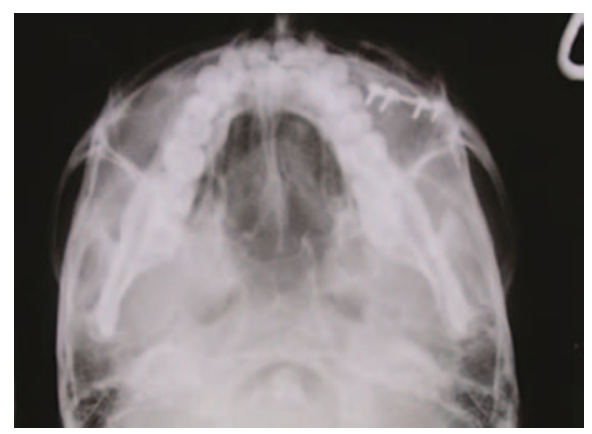

Fig. 3 Showing post-operative X-ray

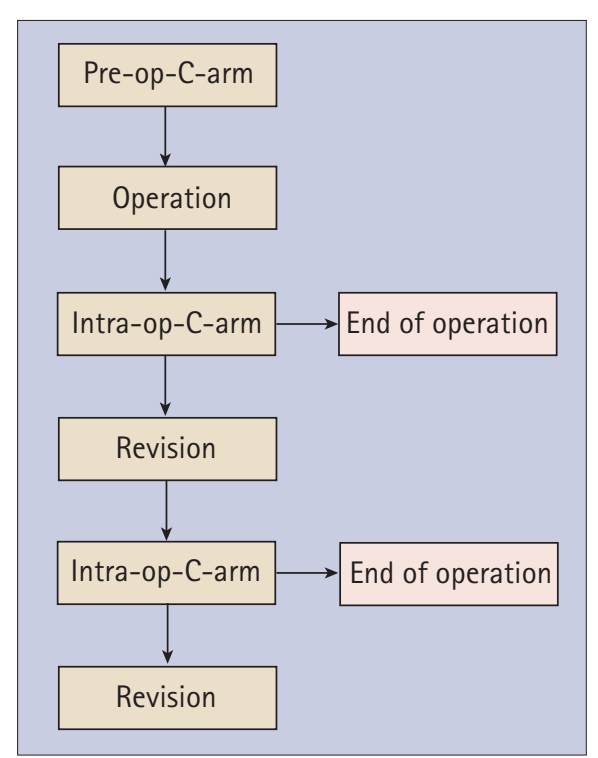

Fig. 4 Algorithm for use of C-Arm

precise reduction is not possible. CT Scan is considered gold standard in the diagnosis of zygomatic arch fractures. However, its intraoperative use for the assessment of reduction of zygomatic arch requires a special operating table and is not cost effective.

The authors used portable C-Arm and recommend the algorithm shown in Figure 4 for intraoperative assessment of fracture reduction in isolated cases of zygomatic arch fracture, or associated with minimally displaced zygomaticomaxillary complex fracture. C-Arm is a truly flexible system offering outstanding levels of performance for a wide range of applications: it is used in routine trauma surgery, orthopaedics, etc. It offers a choice of two image intensifier sizes, evolutive memory capacities and a wide range of options (printers, VCR, laser system, dose measurement). C-Arm system is so called because the $\mathrm{X}$-ray tube support is shaped like a ' $\mathrm{C}$ '. At one end of the ' $\mathrm{C}$ ' is the $\mathrm{X}$-ray tube and at the other end of the ' $\mathrm{C}$ ' is the image receptor. They are mainly automated and have foot pedals to control most operations. This makes them ideal for direct use by surgeons eliminating the need for further personnel. There are therefore considerable logistical and manpower advantages to their use.

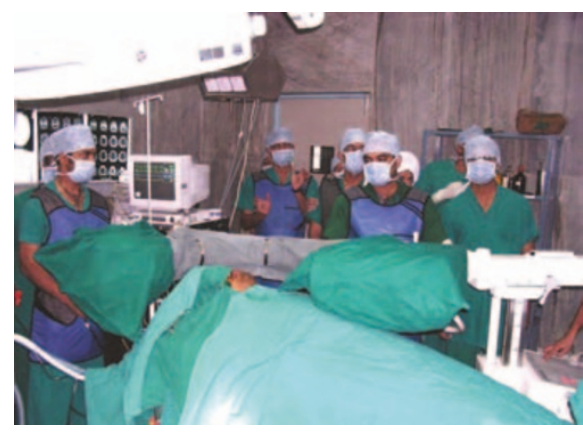

Fig. 5 Showing final position of patient and C-Arm

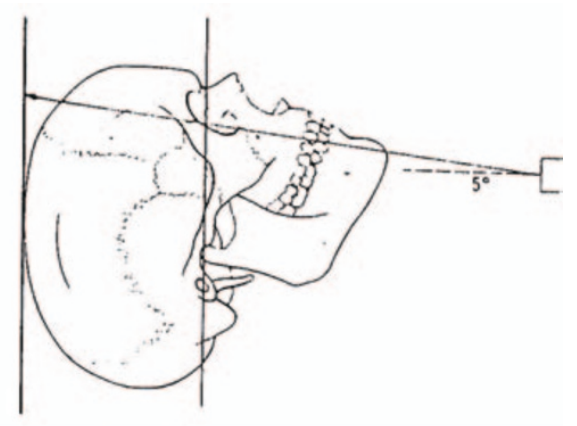

Fig. 6 Showing direction of X-ray beam

\section{The C-Arm Technique}

The patient is positioned in the anti-Trendelenburg position with their neck extended and supported. The C-Arm is positioned such that the X-ray tube is just over $30 \mathrm{~cm}$ (30 $\mathrm{cm}$ is the minimum according to British law) away from the patient in the submental area. The image intensifier is placed as close as possible to the patient in the vertex area (Fig. 5). The X-ray source should be kept as far from the patient as possible and the intensifier must be kept as close as possible. This will reduce the entrance surface dose to the patient and reduce magnification of the image. $\mathrm{X}$-rays are directed from the centre of the chin towards the vertex area approximately $5^{\circ}$ upwards to the imaginary line drawn from menton to bregma (Fig. 6). Usually exposure is done on automatic pulsed fluoroscopy mode. This lowers the exposure when compared with conventional fluoroscopy. If it is not possible to obtain an image of suitable quality from the automatic exposure, a manual override is available. The IRMER [Ionising Radiation (Medical Exposure) Regulations 2000] ${ }^{6}$ regulations state that the manual facility should only be used when justified and experience has shown such occasions to be rare.

Previous dose measurements on staff using the Fluoroscan have shown that monitoring badges are not required if lead aprons are used. This is mainly due to the low scatter produced and the short overall screening times used on most patients. 
Beyond two metres it was found that personal protective equipment was of little benefit and is not recommended. It is, however, important for all people to stay as far away from the beam as is practical during exposures and to ensure that the hands and head of the operator are kept out of the primary beam. The surgeon, scrub nurse and anaesthetist should wear a lead rubber apron within the two-metre zone, but lighter aprons of $0.25 \mathrm{~mm}$ lead equivalent are sufficient. The skirt-andtop-apron format is the best for protection below the surgical table. The Radiation Protection Adviser does not consider that fixed barriers are necessary with mini CArm intensifiers because of the rapid decline in radiation dose with distance as per inverse square law. ${ }^{7}$

\section{CONCLUSION}

We have used C-Arm and found that the use of C-Arm intraoperatively allows for dynamic visualisation of instrumentation and the immediate monitoring of the adequacy of fracture reduction. Two monitors allow comparison of successive images. Moreover, this technique may eliminate the need for postoperative radiograph and CT scan in zygomatic arch fractures.

As C-Arm is safe, gives easily reproducible images and table information without a time lag, it overcomes the disadvantages of radiography and palpation. It has a user-friendly nature and low emission levels. The case presented was successfully treated using C-Arm intraoperatively, suggesting that it is a useful tool in the surgical reduction of the fracture of the zygomatic arch.
1. Guven 0. Stabilisation of the delayed zygomatic arch fracture. Int J Oral Maxillofac. Surg 1987; 16: 445-447.

2. Killey H C. Fractures of the middle third facial skeleton. Bristol: John Wright \& Sons Ltd, 1965.

3. Kruger E. Oral and maxillofacial traumatology. Vol. 2. p 174. Surrey: Quintessence Publishing, 1986

4. Akizuki H, Yoshida H, Michi K. Ultrasonographic evaluation during reduction of zygomatic arch fractures. J Craniomaxillofac Surg 1990; 18: 263-266.

5. Hoelzle F, Klein M, Schwerdtner 0 , etal. Intraoperative computed tomography with the mobile CT Tomoscan M during surgical treatment of orbital fractures. Int J Oral Maxillofac Surg 2001; 30: 26-31.

6. lonising Radiation (Medical Exposure) Regulations 2000. SI Health and Safety 2000 No. 1059.

7. Sinha S, Evans S J, Arundell M K, Burke F D. Radiation protection issues with the use of mini CArm image intensifiers in surgery in the upper limb: Optimisation of practice and the impact of new regulations. J Bone Joint Surg (British Volume) 2004; 86: 333-336. 